His final conclusion is that there is a strong correlation between increasing age and the incidence of fatal cerebrovascular disease, which has not been affected by improved standards of medical care over the past century. It appears that strokes are an inevitable consequence of growing old, and that they can be prevented only by stopping the process of ageing itself. This pessimistic view raises problems about the significanc's for man of research on ageing in animals, in which both the time scale and the ultimate causes of death are so different from human exporience.

Kurtzke has written an instructive monograph. The very helpful summaries of each part have been collected into an extra chapter which provides a concise guide to his individual and intriguing work. It is clearly arranged and well documented with more than 400 references and 86 tables.

A. D. DAYAN

\section{BEHAVIOUR OF MATERIALS}

\section{Materials Science}

By J. C. Anderson and K. D. Leaver. Pp. 276. (Nelson: London, September 1969.) $40 s$.

THrs book, based on a course given by the authors at Imperial College, represents the most recent attempt to bring between one pair of covers an elementary survey of the behaviour of materials, specifically for engineers. The subject matter of such courses has moved a very long way from the catalogues of casting alloys, descriptive accounts of blast furnaces and the like of a few short years ago: the most important single change is the injection of a good deal of solid state physics, touching most particularly on the electrical and magnetic behaviour of crystalline solids.

The task of formulating such a course is formidable, and it is no wonder that the authors have only partially succeeded. As would be expected in view of their personal expertise, the book is somewhat weighted towards semiconductors and magnetic and dielectric materials. This subject matter is erected on a basis of wave mechanics from the beginning, and this feature is as successful as it is unconventional. Many arbitrary features of more orthodox treatments are avoided, and didactically the authors succeed in anticipating the student's likely points of bewilderment. The treatment of motallic conduction is good (though Figs. 12.2 and 12.5 are incompatible). Tho approach to semiconductors, again, is unconventional, starting from the concept of the orbital rather than from band theory. Brillouin zones are never mentioned. As a very elementary approach it could well be more successful with students than the more usual treatment. The magnetism is a trifle compact; thus the reader would have difficulty in understanding why domains exist. The chapter on dielectrics is a model of clear but economic presentation.

The chapter on mechanical properties is less successful. Macroscopic behaviour is well sketched (though it is a pity that hardness tests are presented in terms, almost, of mavic), but the authors have less feeling for the ways of dislocations. These oscillate between being planar and linear defects, with inevitable loss of clarity when, for instance, dislocation sources are touched on. It is a pity that not more use is made here, and elsewhere, of the concept of bond strength, ably introduced in an earlier chapter. This would have made less likely such misstatements as "it is a relatively simple matter for [atomic] planes to slide over cach other" (page 100), or that longrange order always strengthens an alloy (page 156).

The brief introduction to crystals, though well set out, also suffers from some misstatements. Thus, the unit cell of diamond does not contain 12 atoms (page 78); there are 230 space-groups, not crystal classes (page 90); Fig. $7 \cdot 16$ does not portray a twinning shear and the schematic picture of a vitreous silica network (Fig. 10·6) is incorrect.

The chapter on polymers is an excellent introduction, a welcome innovation in a book addressed to other than chemical engineers. The treatment of alloys and ceramics is a moderately successful condensation of a large field, though the treatment of steels is really too concise to be useful (one can hardly hope to understand the point of tempering if martensite is never mentioned), and the concepts of metastability and approach to equilibrium, so central to this field, could only be appreciated if diffusion had been introduced.

The most serious obstacle to understanding the really erueial concept of phase equilibria, however, lies in the inadequate troatment of free energy and entropy (pages 138-140). The presentation of this subtle but vital area contains too many inaccuracies and short cuts to be at all helpful to the student (to pick just one example, maximization of entropy is presented as a universal principle, without specifying an isolated system). Without a propor grounding here, phase equilibria are bound to appear as though pulled out of a hat, and the fact that vacancios, unlike dislocations, are stable defects is incomprehensible.

The inadequacy of this section makes it impossible to agree with the authors that the book will be of assistance to students of chemistry or metallurgy, but it should bo very successful at an elementary level for electrical and electronic engineers and may, with rather more provisos, be so for mechanical and civil engineers also.

R. W. CAHN

\section{TECHNOLOGY OF SOUND}

\section{Physical Foundations of Technical Acoustics}

By I. Malocki. Translated by Irona Bellert. Pp. xxv + 743. (Pergamon: Oxford, London and New York; PWN - Polish Scientific Publishers: Warsaw, Scptember 1969.) 220s; $\$ 28$.

Acoustical technology is undergoing rapid development at the present time; the acoustical engineer has to contend with a larger ratio of extreme frequencies than in almost any other technology, and his devices frequently have to operate satisfactorily with wavelengths both larger and smaller than their physical dimensions at the same time. A book which has as its declared aim the presentation of the physical meaning and mathematical expression of phenomena, to aid those engaged in acoustical engineering, is therefore very wolcome.

It must be said, however, that this aim is fulfilled only in part, as the book is principally a study of acoustic wave propagation. It is well done, with a carefully organized treatment, which progresses from the ideal medium to the properties of real fluids and solids. The mathematical level is high, although not is high as in some theoretical textbooks; it is clearly presented, and the treatment shows the approach of a physicist. Experimental results are discussed in some chapters; but there is no mention of developments such as the interaction of sound with light.

Non-linear vibrations and waves are covered, but the section on vibrating systems and analogies is disappointingly brief; there is no troatment of duality in circuits, or of transducers. Expansion of this section would be of great value, not only for the designer of acoustical devices, but also for the experimonter concerned about the porformance of his apparatus.

Fach chapter has a brief historical introduction, and each has that most useful, although not very common, feature of a number of worked examples (in which, however, different units jostle each other somewhat disconcertingly). References are full, and include many from outside western Europe and the United States; but the index is sketchy and should be much longer. Certain non-standard terms - presumably produced by too literal 